\title{
Nonsequential Double Ionization at the Single-Optical-Cycle Limit
}

\author{
X. Liu, ${ }^{1}$ H. Rottke, ${ }^{1}$ E. Eremina, ${ }^{1}$ W. Sandner, ${ }^{1}$ E. Goulielmakis, ${ }^{2}$ K. O. Keeffe, ${ }^{2}$ M. Lezius, ${ }^{2}$ F. Krausz, ${ }^{2,3}$ F. Lindner, ${ }^{3}$ \\ M. G. Schätzel, ${ }^{3}$ G. G. Paulus, ${ }^{4,5}$ and H. Walther ${ }^{3,4}$ \\ ${ }^{1}$ Max-Born-Institut, Max-Born-Strasse 2a, D-12489 Berlin, Germany \\ ${ }^{2}$ Institut für Photonik, Technische Universität Wien, Gusshausstrasse 27, A-1040 Wien, Austria \\ ${ }^{3}$ Max-Planck-Institut für Quantenoptik, Hans-Kopfermann-Strasse 1, D-85748 Garching, Germany \\ ${ }^{4}$ Ludwig-Maximilians-Universität München, Am Coulombwall 1, D-85748 Garching, Germany \\ ${ }^{5}$ Department of Physics, Texas A\&M University, College Station, Texas 77843-4242, USA
}

(Received 15 June 2004; published 20 December 2004)

\begin{abstract}
We report differential measurements of $\mathrm{Ar}^{++}$ion momentum distributions from nonsequential double ionization in phase-stabilized few-cycle laser pulses. The distributions depend strongly on the carrierenvelope (CE) phase. Via control over the CE phase one is able to direct the nonsequential doubleionization dynamics. Data analysis through a classical model calculation reveals that the influence of the optical phase enters via (i) the cycle dependent electric field ionization rate, (ii) the electron recollision time, and (iii) the accessible phase space for inelastic collisions. Our model indicates that the combination of these effects allows a look into single cycle dynamics already for few-cycle pulses.
\end{abstract}

DOI: 10.1103/PhysRevLett.93.263001

PACS numbers: $33.80 . \mathrm{Rv}, 33.80 . \mathrm{Wz}$

Recent advances in femtosecond laser technology have opened the door to unprecedented insight into intense laser-matter interaction (up to $100 \mathrm{GW}$ ) on the time scale of a few optical cycles [1]. For such few-cycle pulses, the electric field $E(t)=E_{0}(t) \cos (\omega t+\varphi)$ depends strongly on the phase of the carrier wave (having frequency $\omega / 2 \pi)$ with respect to the pulse envelope $E_{0}(t)$, the socalled carrier-envelope (CE) phase $\varphi$ [2]. Since the pioneering work of [3] feedback control of this CE phase has been established [4] and a direct measurement of $\varphi$ was demonstrated recently [5,6]. Today, the spatiotemporal variation of electromagnetic fields consisting of very few cycles is not only precisely known, but it can be shaped with attosecond precision via control over $\varphi$. Because of the exponential dependence of atomic tunnel ionization on the instantaneous electric field strength, control over $\varphi$ has opened the opportunity to define the moment of ionization with a precision of several hundred attoseconds $[7,8]$. The trajectory of an electron born within this narrow time window remains subject to the laser field, which can, in principle, be tailored at will. This allows for control over various rescattering processes of the field liberated electron on the parent ion. Indeed, it has been found that for processes such as high harmonic generation (HHG) [5] and high-order above threshold ionization (ATI) [6] the experimentally observed outcomes are strongly dependent on $\varphi$. A third rescattering mechanism of paticular interest is present in nonsequential double ionization (NSDI) $[9,10]$. All the processes introduced here occur when a field accelerated electron returns and hits the parent ion it left before. The collision mechanisms can be classified as recombination (HHG), elastic (ATI), or inelastic (NSDI). The NSDI inelastic case causes dislodging of a second electron from the ion core and thus represents an excellent example for a heavily perturbed three-body Coulomb sys- tem. Similar to HHG and ATI, a significant sensitivity on $\varphi$ has already been predicted for the NSDI final state electron-electron momentum correlation [11]. This indicates the possibility to steer double ionization efficiently using $\varphi$. In this Letter we present the first experimental evidence that control over $\varphi$ is indeed a suitable means to direct nonsequential double-ionization dynamics. Our data demonstrate a strong CE phase sensitivity of the differential momentum distribution of $\mathrm{Ar}^{2+}$ ions along the laser beam polarization axis. Moreover, our model calculation indicates that we are able to look into single cycle dynamics of NSDI even though the light pulses still consist of several cycles.

The experimental technique is based on cold target recoil ion momentum spectroscopy (COLTRIMS) [12]. Details of our experimental setup can be found in $[13,14]$. Briefly, a low-density $\left(10^{8}\right.$ atoms $\left./ \mathrm{cm}^{3}\right)$ supersonic argon atomic beam is intersected by a laser beam at its focal spot. A weak electric field $\left(1-7 \mathrm{~V} \mathrm{~cm}^{-1}\right)$ extracts the ions created in the focus. Particles are recorded by a microchannel plate detector, and their initial momenta are determined from the measured time of flight and the position where they hit the detector. The solid angle of ion detection is $4 \pi$. Laser pulses are obtained from a commercial femtosecond oscillator and amplifier (Femtolasers $\mathrm{GmbH}$ ) followed by a hollow fiber pulse compressor. $500 \mu \mathrm{J}$ pulse energy and $5 \mathrm{fs}$ pulse width are produced at a center wavelength of $760 \mathrm{~nm}$. The CE phase is stabilized using a three-stage servo loop. The first and second stages stabilize the phase of the oscillator and amplifier, respectively [5]. Both employ the self-referencing scheme known from optical frequency metrology [3]. Long-term phase stability (the measurements presented below required hours of data acquisition) is accomplished with the stereo-ATI method $[6,15]$ by recording spectra of pho- 
toelectrons emitted in opposite directions. These exhibit characteristic phase effects. Besides its use as a phase meter, the stereo-ATI helps in monitoring the laser performance. The experimental setup is sketched in Fig. 1. A small fraction $(5 \%)$ of the laser radiation is rerouted to the stereo-ATI apparatus by use of a beam splitter. Precise adjustment of the amount of glass (W2) in the beam path corrects for the difference in optical path lengths to the stereo-ATI and to the COLTRIMS apparatus. This ensures short pulses in both experiments simultaneously. Control of W2, however, does not imply identical values of $\varphi$; in fact, the offset $\Delta \varphi=\varphi_{\text {ATI }}-\varphi_{\text {COLTRIMS }}$ as well as $\varphi_{\text {COLTRIMS }}$ itself is unknown during the experiment. However, what matters is that $\Delta \varphi$ is constant. In order to actively control the CE phase, the pair of glass wedges (W1) in the common beam path is equipped with a stepper motor and automatically controlled by the ATI phase meter. Hence, during the whole measurement the CE phase $\varphi_{\text {ATI }}$ is measured and kept at a fixed value. In order to scan different values of $\varphi_{\text {COLTRIMS }}$, the amount of glass of W2 and therefore $\Delta \varphi$ is changed by known amounts. An addition of $26 \mu \mathrm{m}$ of fused silica into the beam path changes $\varphi$ by $\pi$.

Figure 2 shows $\mathrm{Ar}^{++}$ion momentum $\left(P_{\|}\right)$distributions along the laser polarization direction for different $\mathrm{CE}$ phases measured at a fixed laser pulse width $(\approx 5 \mathrm{fs})$ and intensity $\left(\approx 350 \mathrm{TW} / \mathrm{cm}^{2}\right)$. The ion momentum resolution is 0.1 a.u. The spectra reflect the distributions of the corresponding sum-momentum component of the two photoelectrons [16]. A prominent asymmetric pattern is visible which changes with the CE phase. A nearly symmetric shape is found only at one setting of the phase $\left(\varphi=\varphi_{0}\right)$. Switching off the phase stabilization of the laser gives rise to a symmetric ion momentum distribution [Fig. 2(f)]. It closely resembles "long" pulse spectra at light intensities where double ionization of Ar proceeds predominantly

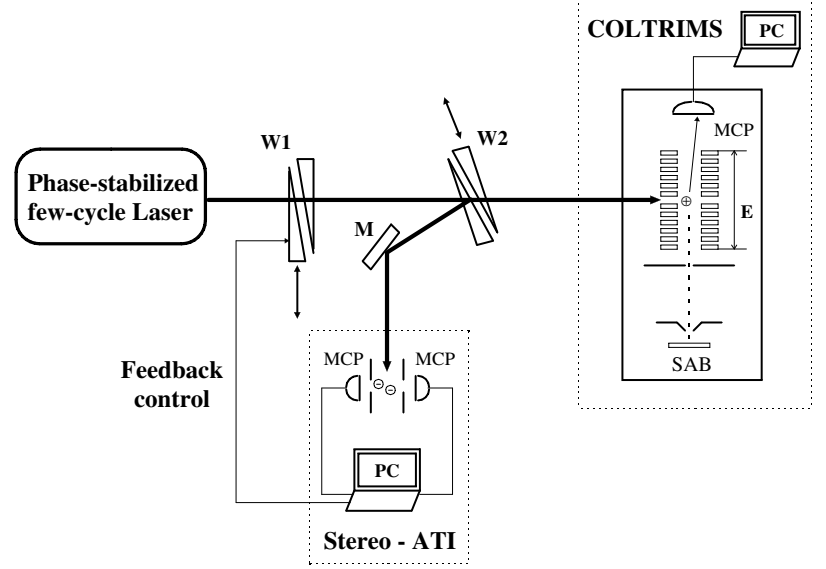

FIG. 1. Schematic view of the experimental setup. W1, W2: two pairs of glass wedges; MCP: position sensitive microchannel plate detector; M: mirror; E: extraction electric field; SAB: supersonic atomic beam. nonsequential $[13,16]$. This, the observed strong phase sensitivity of the spectra, and our model calculation (see below) clearly indicate that nonsequential double ionization of Ar also prevails in the few optical cycle regime. Moreover, sequential double ionization with both electrons leaving the atom uncorrelated should show a similar phase dependence as single ionization, where integrated phase effects have been found to be of the order of only $10 \%$ [6]. This is much less than we find here for double ionization. The interesting points to be noted in Fig. 2 are as follows: (i) The fixed phase symmetric distribution $\left(\varphi=\varphi_{0}\right)$ is slightly narrower than the distribution measured with the free-running phase at the same light intensity. (ii) The minimum in the $\mathrm{Ar}^{++}$yield at $P_{\|}=0$ is more pronounced in the fixed phase spectrum. (iii) A phase change of $\pi$ transforms the momentum distribution into its mirror image with respect to reflection at $P_{\|}=0$ [compare Figs. 2(a) -2 (d) and 2(b) $-2(e)]$.

In order to understand how the $\mathrm{CE}$ phase steers the ion momentum distribution and to determine $\varphi_{0}$, a classical model based on the light induced recollison mechanism of NSDI is applied to simulate the experimental results. This simple model has already been used successfully to analyze NSDI of molecules ionized by long laser pulses [14]. It is in remarkably good agreement with a more refined quantum mechanical calculation [17]. The idea behind our model is that NSDI is caused by an $(e, 2 e)$ process induced by recollision of an electron on the singly charged ion core after it has been accelerated by the electric field of the light pulse. The recolliding electron is initially set free by field ionization at a time $t_{0}$ and collides with the ion core at a later time $t_{1}$. In turn, the probability for NSDI depends
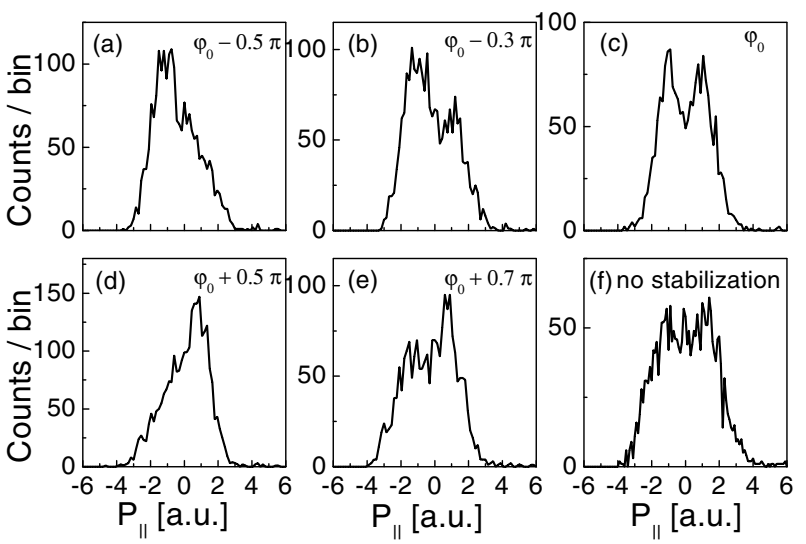

FIG. 2. Experimental momentum distribution of $\mathrm{Ar}^{2+}$ ions from NSDI of $\mathrm{Ar}$ at different $\mathrm{CE}$ phases $\varphi$. The laser pulse parameters are $\approx 5 \mathrm{fs}$ pulse width and $\approx 350 \mathrm{TW} / \mathrm{cm}^{2}$ peak light intensity. The distributions are integrated over the Cartesian momentum components perpendicular to the light beam polarization axis. $\varphi_{0}$, shown on the panels, is determined from comparison with a theoretical model to be $0.2 \pi$. The spectrum in panel (f) was taken with the phase unstabilized at otherwise unchanged experimental parameters. 
critically on the initial electric field ionization rate and on the kinetic energy $E_{\mathrm{kin}}\left(t_{1}\right)$ of the electron at the time of recollision. Both the rate and $E_{\text {kin }}\left(t_{1}\right)$ depend sensitively on $t_{0}$. In our model NSDI is possible only at return energies which exceed the ionization potential of the singly charged ion.

For laser pulses with many cycles, field ionization at $t_{0}$ of an electron which recollides at $t_{1}>t_{0}$ can happen at many oscillation maxima of $E(t)=E_{0}(t) \cos (\omega t+\varphi)$ $(\omega t+\varphi=n \pi$ with $n$ an integer). At subsequent halfcycles $n$ the electric field essentially only changes sign. This gives rise to a symmetric momentum distribution for the doubly charged ions with respect to reflection at $P_{\|}=$ 0 . In contrast, in a few-cycle pulse the amplitude of the electric field envelope changes significantly for successive half-cycles depending on the set CE phase $\varphi$. This is exemplified in the three upper panels of Fig. 3. Accordingly, the probability that an electron enters the continuum at some given time $t_{0}$ becomes sensitive to $\varphi$. This and the change in $E_{0}(t)$ between $t_{0}$ and $t_{1}$ breaks the symmetry for electron trajectories (see also [6]). In addition, the recollision time $t_{1}$ and the kinetic energy $E_{\text {kin }}\left(t_{1}\right)$ of the electron colliding with the ion core are depending on $\varphi$ and will further amplify the left-right asymmetry. The classically accessible phase space for impact ionization by the returning electron is given by $E_{\text {kin }}\left(t_{1}\right) \geq I_{p}^{+}$, where $I_{p}^{+}$ is the ionization potential of the singly charged ion. Whether this threshold is reached depends significantly on successive half-cycles, as can be seen in the lower panel in Fig. 3. At each $t_{0}$ the light gray dotted line gives the maximum accessible momentum $p_{e, \|}$ one electron can aquire after impact ionization, and the black line the minimum momentum. Within every half-cycle the two lines thus characterize the outermost boundary of the classically

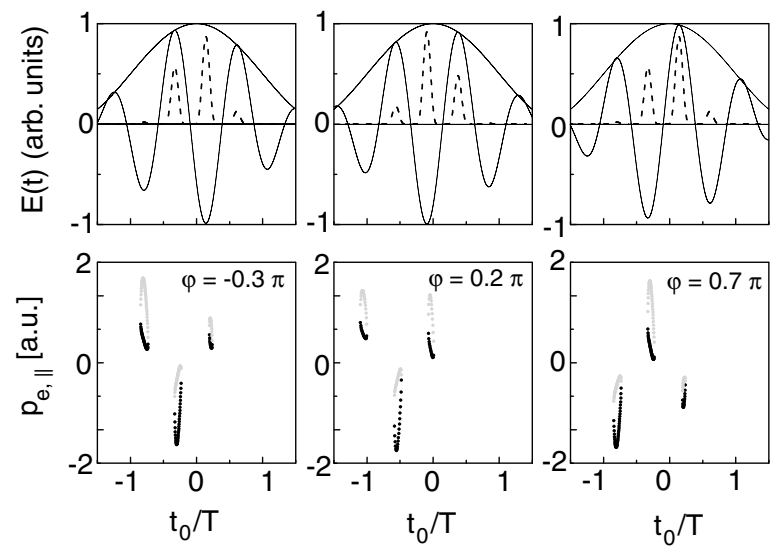

FIG. 3. Upper panel: electric field $E(t)$ (solid line) and the quasistatic field ionization rate [18] (dashed line) for a four-cycle laser pulse $\left(760 \mathrm{~nm}\right.$ center wavelength, $3.5 \times 10^{14} \mathrm{~W} / \mathrm{cm}^{2}$ peak intensity). Lower panel: classically accessible final phase space upon recollision $\left(p_{1 \|}=p_{2 \|}=p_{e, \|}\right)$ for electrons liberated at tunneling time $t_{0}$ (in units of the optical period $T=2.6 \mathrm{fs}$ ). $\varphi$ denotes the corresponding $\mathrm{CE}$ phase. accessible final phase space for the momentum component $P_{\|}=2 p_{e, \|}$ of $\mathrm{Ar}^{++}$[19]. A missing border line in a halfcycle means that for electrons which are field ionized in this cycle $E_{\text {kin }}\left(t_{1}\right)<I_{p}^{+}$upon recollision. The final momentum distribution of the doubly charged ion is determined by the interplay between three factors: the tunnel ionization rate as a function of $t_{0}$, the return time $t_{1}$, and $E_{\text {kin }}\left(t_{1}\right)$. Numerical results for the $\mathrm{Ar}^{++}$momentum distribution are shown in Fig. 4. They are based on the classical model outlined above with an electron-electron contact interaction for the collision which is assumed to happen at the position of the ion core [17]. A gradual change of asymmetry appears with the center of gravity of the distribution shifting from negative to positive ion momentum with an increasing CE phase. A true symmetric distribution is not observed at any CE phase. Only near $\varphi=0.2 \pi$ (and $1.2 \pi$ ) a double hump structure with maxima of nearly equal strengths appears. However, the maxima do not appear at symmetric positions with respect to $P_{\|}=0$, and the humps differ in width. We used the simulation to assign an absolute value of $0.2 \pi$ to $\varphi_{0}$ (see Fig. 2). The accuracy of this assignment is about $\pm 0.1 \pi$. Figure 2(f) demonstrates how sensitive the ion momentum distribution reacts to the width of the laser pulse. For an eight-cycle pulse the CE phase loses any influence on the momentum distribution, even at $\varphi=0.7 \pi$, a value where shorter pulses show the most pronounced asymmetry.

The simulation for $\varphi=-0.3 \pi, 0.7 \pi$ (Figs. 3 and 4) indicates that the major reason for the experimentally observed strong asymmetric ion momentum distributions is the birth of recolliding electrons within only one optical half-cycle $\left(t_{0} / T \approx-0.3 \pi\right.$ for both $\varphi=-0.3 \pi$ and $\varphi=$ $0.7 \pi)$. A "beam" of scattering electrons hits the parent ion mainly from one side. Effective hits from the other side are
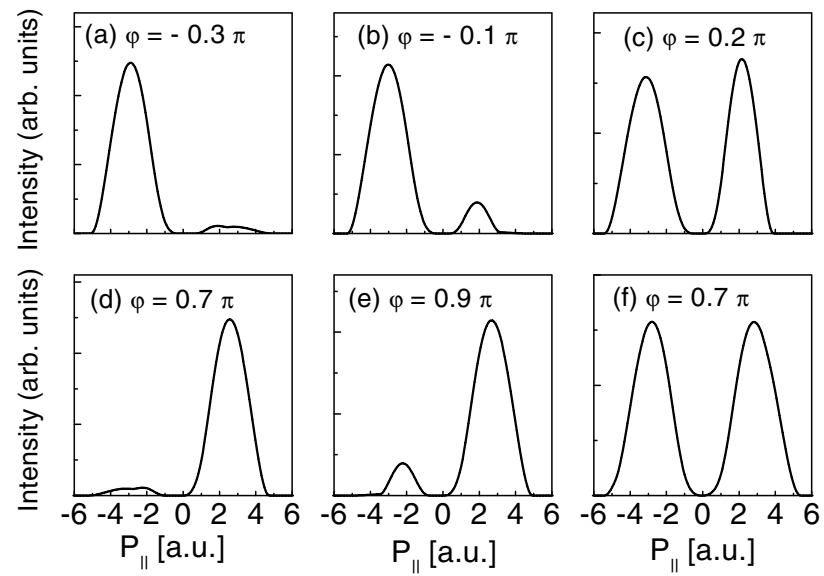

FIG. 4. Numerical simulation of the ion momentum distribution induced by few-cycle pulses at different CE phases. A fourcycle laser pulse is used in the calculation for (a)-(e) and an eight-cycle pulse in (f). The other parameters are the same as in Fig. 3. 
suppressed by the combination of a small electric field ionization rate, and, in addition, lack of accessible phase space for the inelastic collision. Figure $3(\varphi=-0.3 \pi$, $\varphi=0.7 \pi)$ reveals that the inelastically scattering electrons which give rise to the predominating maxima in the corresponding ion momentum distributions in Fig. 4 are even not released during the half-cycle with the highest electric field strength. Regarding the "symmetric" case $(\varphi=0.2 \pi)$ it is observed that two consecutive half-cycles contribute effectively to NSDI. The deviation from full symmetry of the double peak structure in Fig. 4(c) (widths and position of the two maxima) has the following origin: a significantly different accessible phase space and different field ionization rates in the two relevant half-cycles (see Fig. 3).

Overall we find the agreement between this simple model and our experimental results encouraging, even if a closer comparison yields some discrepancies. (i) The calculated yield of $\mathrm{Ar}^{++}$ions with momentum $P_{\|} \approx 0$ is always close to zero, whereas the yield found in the experiment is significantly higher. Moreover, (ii) at $\varphi=$ $-0.3 \pi$ and at $\varphi=0.7 \pi$ nearly all ions are found with either $P_{\|}>0$ or $P_{\|}<0$ in the calculation. The experiment shows distributions with a clear "hanging shoulder" at these phases but a significant amount of ions is always found having an opposite momentum. In this respect it is important to note that a second significant NSDI channel exists for Ar. It is assumed to be the impact excitation of $\mathrm{Ar}^{+}$into bound excited states with subsequent electric field ionization of the excited electron [20]. This process allows the formation of photoelectron pairs with similar momenta emitted in opposing directions, giving rise to $\mathrm{Ar}^{++}$ions with $P_{\|} \approx 0$. In our model this channel has not been incorporated. To learn more about the influence of this ionization channel it will be necessary to determine the final state electron momentum correlation [20]. (iii) The separation of the maxima in the experiment is smaller than that in the calculated spectra. However, the three-body contact interaction used here to describe the inelastic collision is known to result in a momentum separation usually larger than observed experimentally [21].

In conclusion, our experimental study shows that the ion momentum distribution following NSDI in few-cycle laser pulses reacts very sensitively to the $\mathrm{CE}$ phase $\varphi$. Consequently, via control over $\varphi$ one is able to direct nonsequential double-ionization dynamics. The analysis of the experimental data through a classical model calculation reveals that the influence of the optical phase enters via (i) the cycle dependent electric field ionization rate, (ii) the electron recollision time, and (iii) the accessible phase space for inelastic collisions. It also shows that the combination of these effects allows a look into single cycle dynamics already if few-cycle pulses are used, since, de- pending on the phase, only one or at most two optical halfcycles effectively contribute to NSDI. Being able to steer NSDI by control over the recolliding electrons via the CE phase goes well beyond NSDI itself. As proposed recently [7], recolliding electrons may be used as an effective probe for the attosecond time domain, comparable to the presently used attosecond extreme ultraviolet pulses [8]. Our work now demonstrates that it is possible to control electrons coherently in space and in time via control over the CE phase.

We gratefully acknowledge support by the Deutsche Forschungsgemeinschaft (DFG) and fruitful discussions with W. Becker and C. Figueira de Morisson Faria. E. Goulielmakis and K. O'Keeffe have been supported by the Austrian Science Funds (Grants No. F016, No. P14447, No. P15382, and No. Z63). G. G. Paulus acknowledges support from The Welch Foundation.

[1] M. Nisoli et al., Opt. Lett. 22, 522 (1997); G. Steinmeyer et al., Science 286, 1507 (1999).

[2] T. Brabec and F. Krausz, Rev. Mod. Phys. 72, 545 (2000).

[3] J. Reichert, R. Holzwarth, Th. Udem, and T. W. Hänsch, Opt. Commun. 172, 59 (1999); H. R. Telle et al., Appl. Phys. B 69, 327 (1999).

[4] D. J. Jones et al., Science 288, 635 (2000); A. Apolonski et al., Phys. Rev. Lett. 85, 740 (2000).

[5] A. Baltuška et al., Nature (London) 421, 611 (2003).

[6] G. G. Paulus et al., Phys. Rev. Lett. 91, 253004 (2003).

[7] H. Niikura et al., Nature (London) 417, 917 (2002).

[8] R. Kienberger et al., Nature (London) 427, 817 (2004)

[9] P. B. Corkum, Phys. Rev. Lett. 71, 1994 (1993).

[10] R. Dörner et al., Adv. At. Mol. Opt. Phys. 48, 1 (2002).

[11] X. Liu and C. Figueira de Morisson Faria, Phys. Rev. Lett. 92, 133006 (2004).

[12] J. Ullrich, R. Moshammer, A. Dorn, R. Dörner, L. Ph. Schmidt, and H. Schmidt-Böcking, Rep. Prog. Phys. 66, 1463 (2003).

[13] E. Eremina, et al., J. Phys. B 36, 3269 (2003).

[14] E. Eremina et al., Phys. Rev. Lett. 92, 173001 (2004).

[15] G. G. Paulus et al., Nature (London) 414, 182 (2001).

[16] Th. Weber et al., Phys. Rev. Lett. 84, 443 (2000); R. Moshammer et al., Phys. Rev. Lett. 84, 447 (2000); Th. Weber et al., J. Phys. B 33, L127 (2000).

[17] C. Figueira de Morisson Faria, X. Liu, W. Becker, and H. Schomerus, Phys. Rev. A 69, 021402(R) (2004).

[18] M. V. Ammosov, N. B. Delone, and V.P. Krainov, Zh. Eksp. Teor. Fiz. 91, 2008 (1986) [Sov. Phys. JETP 64, 1191 (1986)].

[19] C. Figueira de Morisson Faria and W. Becker, Laser Phys. 13, 1196 (2003).

[20] B. Feuerstein et al., Phys. Rev. Lett. 87, 043003 (2001).

[21] R. Kopold, W. Becker, H. Rottke, and W. Sandner, Phys. Rev. Lett. 85, 3781 (2000). 